\title{
Medindo a percepção ambiental de universitários com a escala 2-MEV: Validação com a modelagem de equações estruturais e a regressão quantílica
}

Measuring the environmental perception of university students using the 2-MEV scale: Validation with structural equation modeling and quantile regression

Medición de la percepción ambiental de alumnos mediante la escala 2-MEV: Validación con modelado de ecuaciones estructurales y regresión cuantílica

José Francisco dos Reis Neto

ORCID: https://orcid.org/0000-0002-1152-1149

Universidade Anhanguera Uniderp, Brasil E-mail: jose.rneto@anhanguera.com

Celso Correia de Souza

ORCID: https://orcid.org/0000-0002-2689-8264 Universidade Anhanguera Uniderp, Brasil E-mail: csouza939@gmail.com

Pablo Antonio Muñoz-Gallego

ORCID: https://orcid.org/0000-0003-4531-2051 Universidad de Salamanca, Espanha E-mail: pmunoz@usal.es

Taner Douglas Alves Bitencourt ORCID: https://orcid.org/0000-0002-1501-8567 Universidade Anhanguera Uniderp, Brasil

E-mail: taner.bitencourt@anhanguera.com

Davi Guimarães Soares

ORCID: https://orcid.org/0000-0003-0965-3019 Universidade Anhanguera Uniderp, Brasil E-mail: davigsoares@hotmail.com

Patricio Lauro de Melo Neto

ORCID: https://orcid.org/0000-0003-0587-5597 Faculdade de Ciências Sociais Aplicadas de Sinop, Brasil E-mail: patricio.neto@kroton.com.br

\section{Resumo}

Este artigo teve como objetivo avaliar o modelo 2-MEV: Dois Principais Valores Ambientais, como uma escala para a medição de atitudes ambientais de universitários, relacionada à preservação e à utilização da natureza. Para testar o modelo foi coletado uma amostra de 241 universitários, por meio de um questionário estruturado. As declarações das escalas foram validadas utilizando a técnica da análise fatorial confirmatória, aplicando a modelagem de equações estruturais, baseado em covariâncias. Os escores dos dois fatores de preservação e utilização da escala 2-MEV foram mapeados em quadrantes ortogonais, seguindo de uma análise de regressão quantílica para estabelecer a distribuição condicional linear dos dois fatores em cada quartis. Os resultados indicaram uma validação e consistência da escala 2MEV na aplicação aos universitários, indicando que eles são na sua maioria tem atitude biocêntrica. As mulheres e os universitários da área de conhecimento da Saúde revelaram atitudes mais biocêntricos que as demais categorias. Os resultados analíticos das regressões quantílicas, para os quartis de $25 \%$ (quartil inferior), 50\% (mediana) e $75 \%$ (quartil superior), demonstraram os coeficientes do fator utilização são negativos, relacionando que um aumento no seu valor absoluto, provoca uma atitude mais antropocêntrica e uma diminuição na atitude biocêntrica. É reconhecido que a amostra por conveniência dos universitários pode ser uma limitação da pesquisa. Recomenda-se que a amostra seja ampliada e que estudos longitudinais ocorram, inclusive com a inserção de programas de educação ambiental. Como os dados e o modelo se mostraram consistentes, este artigo possui sua contribuição no conhecimento acadêmico.

Palavras-chave: Atitudes ambientais; Modelo 2-MEV; Modelagem de equações estruturais; Preservação da natureza; Regressão quantílica.

\section{Abstract}

This article aimed to evaluate the 2-MEV model: Two Major Environmental Value, as a scale for measuring environmental attitudes of university students, related to the preservation and use of nature. To test the model, a 
sample of 241 university students was collected through a structured questionnaire, like a survey. The scale statements were validated using the confirmatory factor analysis technique, applying structural equation modeling, based on covariance. The scores for the two factors of the 2-MEV scale were mapped in orthogonal quadrants, following a quantile regression analysis to establish the linear conditional distribution of the two factors in each quartile. The results indicated a validation and consistency of the 2-MEV scale when applied to university students, indicating that they are mostly biocentric. Women and university students in health knowledge revealed more biocentric attitudes than the other categories. The analytical results of the quantile regressions, for the quartiles of 25\% (lower quartile), $50 \%$ (median) and $75 \%$ (upper quartile), showed the coefficients of the utilization factor are negative, relating that an increase in its absolute value, causes a more anthropocentric attitude and a decrease in the biocentric attitude. It is recognized that the convenience sample of university students may be a limitation of the research. It is recommended that the sample be expanded and that longitudinal studies take place, including the insertion of environmental education programs. As the data and the model proved to be consistent, this article has its contribution to academic knowledge.

Keywords: Environmental attitudes; 2-MEV model; Structural equation modeling; Nature preservation; Quantile regression.

\section{Resumen}

Este artículo tuvo como objetivo evaluar el modelo 2-MEV: Dos valores ambientales principales, como una escala para medir las actitudes ambientales de los estudiantes universitarios, relacionadas con la preservación y uso de la naturaleza. Para probar el modelo, se recogió una muestra de 241 estudiantes universitarios a través de un cuestionario estructurado. Las declaraciones de la escala se validaron mediante la técnica de análisis factorial confirmatorio, aplicando modelos de ecuaciones estructurales, basados en la covarianza. Los puntajes para los dos factores de preservación y el uso de la escala 2-MEV se mapearon en cuadrantes ortogonales, luego de un análisis de regresión de cuantiles para establecer la distribución condicional lineal de los dos factores en cada cuartil. Los resultados indicaron una validación y consistencia de la escala 2-MEV cuando se aplica a alumnos universitarios, lo que indica que son en su mayoría biocéntricos. Las mujeres y los estudiantes universitarios del área de conocimiento en salud revelaron actitudes más biocéntricas que las demás categorías. Los resultados analíticos de las regresiones cuantílicas, para los cuartiles de $25 \%$ (cuartil inferior), 50\% (mediana) y 75\% (cuartil superior), mostraron que los coeficientes del factor de utilización son negativos, relacionando que un aumento en su valor absoluto provoca una actitud más antropocéntrica y una disminución de la actitud biocéntrica. Se reconoce que la muestra de conveniencia de estudiantes universitarios puede ser una limitación de la investigación. Se recomienda ampliar la muestra y realizar estudios longitudinales, incluida la inserción de programas de educación ambiental. Como los datos y el modelo demostraron ser consistentes, este artículo tiene su contribución al conocimiento académico.

Palabras clave: Actitudes ambientales; Modelo 2-MEV; Modelos de ecuaciones estructurales; Preservación de la naturaleza; Regresión cuantílica.

\section{Introdução}

O meio ambiente tem sofrido modificações decorrentes da demanda do crescimento e do desenvolvimento econômico. A questão de uso local do ambiente, tem impulsionado uma força global, para a sua preservação. Foley et al. (2005), descreveram as mudanças ocasionadas pelo uso do meio ambiente, ampliando o jeito do aproveitamento das florestas, da água e do ar, estimuladas pela necessidade de fornecer alimentos, fibras, água e abrigos para a população mundial. A reação para esse impulso de crescimento, provocou a ampliação das terras agrícolas, em termos de pastagem e lavoura, como também na expansão das áreas urbanas. Houve um aumento no consumo de energia, água, fertilizantes e de resíduos, com a perda da biodiversidade. Geralmente, o uso da terra é considerado uma questão ambiental local, mas está se tornando uma força de importância global. Essas mudanças no uso da terra permitiram que os humanos se apropriassem de uma parcela cada vez maior dos recursos do planeta, mas também minam potencialmente a capacidade dos ecossistemas de sustentar a produção de alimentos, manter os recursos de água doce, das áreas florestais, manutenção da biodiversidade, regular o clima e a qualidade do ar, e diminuir a dissipação de doenças. Usar ou não usar o meio ambiente para atender as necessidades humanas é um ponto importante do gerenciamento das capacidades da biosfera em fornecer bens e serviços, ao longo dos tempos (Boucher, Arseneault, Sirois, \& Blais, 2009). As pessoas devem entender que o desenvolvimento sustentável é a conscientização de sua responsabilidade em desacelerar ou mesmo economizar a exploração da biodiversidade.

Mais recentemente, foram apresentados definições e entendimentos mais relacionais ao desenvolvimento sustentável 
(Mikhailova, 2004; Sachs, 2008; Nossa, Rodrigues, \& Nossa, 2017). O conceito de desenvolvimento sustentável passa ser entendido como dimensões integradas do meio ambiente, social, econômica, política, cultural e territorial (Sachs, 2008). Costa, Neumann, Dorion, Olea, \& Severo (2019), relatam que a incorporação dos interesses e das responsabilidades com o meio ambiente, envolvem atitudes ambientalistas dos grupos de interesses à sustentabilidade.

Os argumentos apresentados por Johnson e Manoli (2011), discutem que o conhecimento da situação ambiental não é suficiente para mudanças de comportamento, mas o mais importante de determinação do comportamento é a atitude. Eles seguem com a opinião de que o relacionamento entre a atitude e o comportamento é proporcionado pela educação.

Ao revisitar a teoria do comportamento planejado, Ajzen (2020), descreve que a atitude em relação ao comportamento é considerada como uma crença. Desta forma, a crença comportamental é a probabilidade subjetiva da pessoa realizar o seu comportamento pessoal de interesse. Em estudo anterior, Ajzen e Fishbein (1977), forneceram que a atitude pode ser medida por quatro elementos diferentes: a ação, o alvo para o qual a ação é dirigida, o contexto em que a ação é realizada e o momento em que é realizada. A generalidade ou especificidade de cada elemento depende do procedimento de medição empregado. Desta forma, existem fortes evidências que suportam uma relação causal entre a atitude e o comportamento, se medições apropriadas são usadas para ambas as componentes.

A relação dos universitários com a natureza é considerada de grande importância. Já que, considerando a conhecimento e habilidade adquiridas nas disciplinas acadêmicas, a sua relação de atitudes e comportamentos ambientais podem ser importantes para a sociedade e preservação da natureza, como fator cumulativo e disseminador. Consequentemente, conhecer os valores ambientais em linha com o conhecimento relacionado, deste segmento da sociedade, é uma das formas de avaliar a atitude ao meio ambiente.

A literatura acadêmica indica vários esforços para medições de atitudes ambientais, podendo citar, por exemplo, o Novo Paradigma Ambiental (Dunlap \& Van Liere, 1978), Novo Paradigma Ecológico (Dunlap, Van Liere, Mertig, \& Jones, 2000), Visão Ecológica Mundial (Blaikie, 1992), 2-MEV: Dois Principais Valores Ambientais (Bogner \& Wiseman, 2006), EAATSD: Atitudes Ecocêntricas e Antropocêntricas para o Desenvolvimento Sustentável (Kopnina, 2013).

Embora existam várias escalas de medições de atitudes e comportamentos ambientais, este artigo destaca a proposta de Bogner e Wiserma (2006) nomeada de Dois Principais Valores Ambientais (2-MEV). Com o suporte teórico, a escala de medição foi projetada para medir dois fatores de atitudes ortogonais, os quais receberam a denominação de valor de Preservação (PRE) e de Utilização (UTL). Bogner (2018) descreve que a escala 2-MEV foi amplamente aplicada em várias situações, apresentando suportar os bons indicadores de validação para o modelo ortogonal, em adultos e adolescentes em diferentes países. Inicialmente, a escala 2-MEV foi construída com dez itens para o fator PRE e outros dez itens para o fator UTL. Com o desenvolvimento e aplicações, o número de itens para o fator PRE passou para nove, e para o fator UTL para sete (Johnson \& Manoli, 2011; Manoli, Johnson, Buxner, \& Bogner, 2019), cuja estrutura foi adotada neste artigo.

A escala 2-MEV consegue diferenciar as pessoas que têm mais atitudes preservacionista (PRE, biocêntrica) daquelas com mais atitudes de utilização ambiental (UTL, antropocêntrica). A escala PRE com alta pontuação reflete uma preferência positiva de conservação ambiental, e alta pontuação em UTL indica uma maior utilização e exploração da natureza. Como modelo é ortogonal entre PRE e UTL, permite que a pessoa possa expressar o seu apoio à preservação (PRE) e à utilização (UTL), de forma que conflitos individuais entre a proteção da natureza e o uso dos recursos naturais não existam (Bogner \& Wiseman, 2006). Estes dois fatores da escala 2-MEV permitem que os indivíduos sejam colocados em um dos quatro quadrantes ao invés de classificá-los em uma medida contínua. Considerando o ponto médio da escala de Likert de 7 pontos utilizada (descrita na seção de Metodologia) e o estabelecimento de quadrantes, para um alto escore de PRE (>4) e um baixo escore de UTL (<4) pode significar um indivíduo mais ambientalista (PRE+/UTL-), com atitudes para a conservação da natureza. Um baixo escore de PRE $(<4)$ e um alto escore de UTL $(>4)$ pode significar que o indivíduo possui uma apatia 
frente à conservação e sente que os recursos naturais devem ser usados em benefício ao desenvolvimento humano (PRE(UTL+). Com a mesma lógica de pontuação, os indivíduos com altas pontuações em PRE e em UTL, no quadrante (PRE+/UTL+), indicam um forte compromisso com a conservação da natureza e, ao mesmo tempo, acreditam que ela deve servir em benefício do desenvolvimento das pessoas. Também, indivíduos com baixas pontuações em PRE e em UTL, localizados no quadrante (PRE-/UTL-), podem indicar uma falta de interesse no assunto relacionado à natureza.

O propósito deste artigo foi examinar a aplicação da escala bidimensional 2-MEV em universitários de uma Instituição de Ensino Superior (IES). Três objetivos foram considerados: 1) obter os fatores da 2-MEV, preservação e utilização, com base nos dados coletados junto aos universitários; 2) realizar uma análise fatorial confirmatória do modelo proposto por Manoli et al. (2019); 3) com base nos escores dos fatores, localizar os respondentes nos quatro quadrantes; e 4) avaliar as características demográficas dos acadêmicos em relação à preservação (PRE) e à utilização (UTL).

\section{Metodologia}

A pesquisa deste estudo foi classificada como quantitativa, transversal, com o levantamento de referências publicadas na literatura acadêmica, empregando o procedimento survey para a obtenção dos dados. Os dados foram coletados com um questionário estruturado, aplicado numa amostra por conveniência e não probabilística, proporcionando a possibilita da generalização de aspectos encontrados, significativos e representativos, da população objeto. Os dados quantitativos permitem a utilização de técnicas estatísticas para a validação do modelo e dos resultados encontrados (Gerhardt \& Silveira, 2009; Pereira, Shitsuka, Parreira, \& Shitsuka, 2018).

Para coleta de dados necessários para testar o modelo de Manoli et al. (2019), foi elaborado e empregado um questionário dividido em seções. Na primeira seção, coletou-se os dados do perfil demográfico dos acadêmicos respondentes, quanto: sexo, idade, curso acadêmico, semestre em que está cursando e o período do curso. Na segunda seção, foram apresentadas as nove declarações dos itens da variável PRE, as setes declarações dos itens da variável UTL. Nesta seção, as declarações foram traduzidas do inglês para o português, e avaliadas por três professores da área de meio ambiente, quanto a qualidade da tradução, ajuste de entendimento e proposta de modificações no instrumento final. Os itens das declarações foram medidos mediante uma escala de Likert de concordância, de 7 pontos, codificadas entre $1=$ discordo totalmente, $4=$ não concordo e nem discordo, e 7= concordo totalmente.

Uma amostra intencional e transversal de acadêmicos de graduação presencial em Campo Grande, MS, foi selecionada em uma IES, durante o mês de maio de 2019. As áreas do conhecimento foram: agrárias (agronomia e medicina veterinária), exatas (matemática, engenharias ambiental, civil, computação e elétrica) e saúde (biomedicina, enfermagem e nutrição). Os acadêmicos autopreencheram o questionário, instruídos apenas pelo objetivo da pesquisa, não sendo detalhado os conceitos e definições, esperando que os resultados para a escala 2-MEV surgissem de forma espontânea, do seu domínio ambiental e cultural. Foram coletados 256 questionários, sendo auditados quanto aos dados faltantes ou atípicos, seguindo o procedimento preconizado por Hair Jr et al. (2010). No final, foram utilizados 241 questionários completos e incluídos na análise.

O perfil do acadêmico foi analisado empregando as técnicas estatísticas descritivas. As variáveis latentes da PRE e da UTL foram analisadas quanto as suas consistências internas pelo alfa de Cronbach e a estrutura fatorial aplicando a análise fatorial confirmatória (AFC), com a modelagem de equações estruturais baseada em covariâncias (Byrne, 2016). Os parâmetros de ajuste da AFC foram os considerados os propostos em Hair Jr et al. (2010).

Para a avaliação e relação dos perfis dos acadêmicos com os fatores PRE e UTL foram mapeados nos quadrantes e estimados os seus centros de dados.

Para a verificação da influência estatística dos regressores, PRE como dependente e UTL como explicativa, 
empregou-se a regressão quantílica (RQ). Enquanto uma regressão múltipla linear, associada à estimativa pelo método dos mínimos quadrados, apresenta os resultados das médias das distribuições correspondentes ao conjunto de variáveis explicativas, é possível com a RQ calcular várias equações diferentes de regressão, que correspondam aos diversos pontos quantílicos das distribuições (Garson, 2014; Koenker \& Hallock, 2001).

Desta forma, a RQ permite analisar a associação entre a variável de resposta e as variáveis explicativas dentre os diversos quantis da distribuição. Isto proporciona uma melhor visão dos impactos entre as variáveis, mesmo quando não ocorre uma distribuição normal dos dados e com dados atípicos (outliers), pois ela utiliza a totalidade dos dados para estimar os coeficientes angulares dos quantis.

Em resumo, a RQ representa os quantis da distribuição, apropriada quando a distribuição é heterogênea. $\mathrm{O} \tau$-ésimo quantil de $\mathrm{Y}$ é o valor que separa uma proporção $\tau$ de valores no limite inferior da distribuição de $\mathrm{Y}$ e uma proporção $(1-\tau)$ valores no limite superior;

O modelo de regressão quantílica, sob a hipótese de Yi, possui uma distribuição condicional linear de xi, pode ser representada para o $\tau$-ésimo quantil por:

$$
\mathrm{Y}_{\mathrm{i}}=\mathrm{x}_{\mathrm{i}} \beta_{\tau}+\mathrm{e}_{\mathrm{i}}
$$

em que $\beta_{\tau}$ e e $e_{i}$ são, respectivamente, os vetores de parâmetros e os resíduos associados ao $\tau$-ésimo quantil, em outros termos, teremos impactos marginais de $\beta \tau$ diferenciados para cada quantil $\tau$.

A representação genérica do modelo para cada quantil $\tau$ será:

$$
\mathrm{Q}_{\tau}(\mathrm{y} \mid \mathrm{X})=\mathrm{X} \beta_{\tau}
$$

Para os processamentos dos dados, das estatísticas descritivas e inferênciais, da modelagem de equações estruturais e das regressões quantílicas, foram empregados os softwares SPSS v.26 e AMOS v.24.

\section{Resultados e Discussão}

As características dos perfis dos universitários participantes são apresentadas na tabela 1. Os perfis foram condensados em grupos com maior interesse à pesquisa. Os dados apresentados indicam uma segmentação dos grupos equilibrada e oportuna para análise multicategorias.

A amostra com 241 universitários foi avaliada pelo critério do poder do teste com valor mínimo de 0,80 , um efeito de tamanho médio de 0,15 e nível de significância de 0,05, bicaudal (Sarstedt \& Mooi, 2018). Foi usado o aplicativo G*Power 3 (Faul, Erdfelder, Lang, \& Buchener, 2007) para estimar a quantidade a priori de uma amostra de 68 universitários. Com a amostra de 241 universitários foi estimado poder do teste a posteriori, obtendo-se o valor de 0,98 (Memon, et al., 2020), indicando uma amostra mais do que suficiente para as análises propostas. 
Tabela 1 - Perfis dos universitários da amostra da Instituição de Ensino Superior.

\begin{tabular}{|c|c|c|c|c|c|c|c|}
\hline \multirow[b]{3}{*}{ Categorias } & & \multicolumn{4}{|c|}{ Sexo } & \multirow{2}{*}{\multicolumn{2}{|c|}{ Total }} \\
\hline & & \multicolumn{2}{|c|}{ Feminino } & \multicolumn{2}{|c|}{ Masculino } & & \\
\hline & & $\mathrm{n}$ & $\%$ & $\mathrm{n}$ & $\%$ & $\mathrm{n}$ & $\%$ \\
\hline \multirow{4}{*}{$\begin{array}{l}\text { Faixa de idade } \\
\text { (anos) }\end{array}$} & $<19$ & 27 & 30,3 & 37 & 24,3 & 64 & 26,6 \\
\hline & 19 a 22 & 23 & 25,8 & 52 & 34,2 & 75 & 31,1 \\
\hline & 23 a 26 & 21 & 23,6 & 35 & 23,0 & 56 & 23,2 \\
\hline & $>26$ & 18 & 20,2 & 28 & 18,4 & 46 & 19,1 \\
\hline \multirow{3}{*}{$\begin{array}{l}\text { Área do } \\
\text { Conhecimento }\end{array}$} & Agrárias & 28 & 31,5 & 75 & 49,3 & 103 & 42,7 \\
\hline & Exatas & 27 & 30,3 & 69 & 45,4 & 96 & 39,8 \\
\hline & Saúde & 34 & 38,2 & 8 & 5,3 & 42 & 17,4 \\
\hline \multirow[t]{2}{*}{ Semestre } & 1 a 3 & 50 & 56,2 & 85 & 55,9 & 135 & 56,0 \\
\hline & 4 a 10 & 39 & 43,8 & 67 & 44,1 & 106 & 44,0 \\
\hline \multirow[t]{2}{*}{ Período } & Diurno & 44 & 49,4 & 35 & 23,0 & 79 & 32,8 \\
\hline & Noturno & 45 & 50,6 & 117 & 77,0 & 162 & 67,2 \\
\hline
\end{tabular}

Fonte: Autores.

As estatísticas descritivas das médias e desvios padrões dos fatores e itens que compuseram a escala 2-MEV, e da confiabilidade interna das escalas, foram calculadas e apresentadas na Tabela 2. As declarações foram extraídas Manoli, Johnson, Buxner e Bogner (2019).

Tabela 2. Média, desvio padrão e alfa de Cronbach das declarações da escala 2-MEV.

\begin{tabular}{|c|c|c|c|}
\hline Sigla & Declaração do item & $\mathrm{m}$ & $\mathrm{dp}$ \\
\hline PRE & Preservação $(\alpha=0,79)$ & 5,3 & 1,15 \\
\hline PRE1 & Se eu tiver dinheiro sobrando, darei algum para ajudar a proteger a natureza. & 5,0 & 2,03 \\
\hline PRE2 & Para economizar energia, controlo a temperatura do ar-condicionado para um pouco mais alta. & 4,1 & 2,17 \\
\hline PRE3 & Eu gostaria de me sentar perto de um lago e observar as borboletas. & 5,2 & 1,99 \\
\hline PRE4 & Eu ajudaria a levantar dinheiro para proteger a natureza. & 5,2 & 1,85 \\
\hline PRE5 & Eu sempre apago a luz quando não preciso mais dela. & 6,0 & 1,70 \\
\hline PRE6 & Eu gosto de viajar para o campo, longe das cidades. & 5,9 & 1,71 \\
\hline PRE7 & Eu tento dizer às pessoas que a natureza é importante. & 5,8 & 1,49 \\
\hline PRE8 & $\begin{array}{l}\text { Tento economizar água tomando banhos mais curtos ou desligando a água enquanto escovo os } \\
\text { dentes. }\end{array}$ & 5,6 & 1,72 \\
\hline PRE9 & Eu gosto do silêncio da natureza. & 6,1 & 1,58 \\
\hline UTL & Utilização $(\alpha=0,72)$ & 3,2 & 1,20 \\
\hline UTL1 & As pessoas têm o direito de mudar o ambiente (natureza). & 3,0 & 2,07 \\
\hline UTL2 & Construir novas estradas é tão importante que as árvores podem ser derrubadas. & 2,6 & 1,84 \\
\hline UTL3 & Eu gosto mais de um gramado do que um lugar onde as flores crescem por conta própria. & 3,2 & 1,97 \\
\hline UTL4 & Porque os mosquitos vivem em pântanos, devemos drená-los e usá-los para a agricultura. & 3,0 & 1,922 \\
\hline UTL5 & Para alimentar as pessoas, a natureza pode ser removida para cultivar alimentos. & 3,2 & 1,86 \\
\hline UTL6 & As pessoas estabelecem como usar a natureza. & 3,4 & 1,96 \\
\hline UTL7 & As ervas daninhas devem ser eliminadas porque ocupam o espaço das plantas que necessitamos. & 4,0 & 2,09 \\
\hline
\end{tabular}

Legenda: $\mathrm{m}$ - média; dp - desvio padrão; $\alpha$ - alfa de Cronbach.

Fonte: Autores.

A Tabela 2 indicou que a confiabilidade interna das escalas dos constructos, calculada com o emprego do alfa de Cronbach, foram valores superiores a 0,70, considerados como aceitáveis (Hair Jr et al., 2010). A verificação de consistência e validação da escala 2-MEV, foi realizada a análise fatorial confirmatória, com os dois fatores PRE (9 itens) e UTL (7 itens).

A Figura 1 apresenta os valores das cargas fatoriais, erros e correlação, em valores padronizados, para o modelo 
estrutural proposto por Manoli et al. (2019) e processados pelo software AMOS v. 24 (Arbuckle, 2016).

Figura 1 - Modelo estrutural para a análise fatorial confirmatória do 2-MEV.

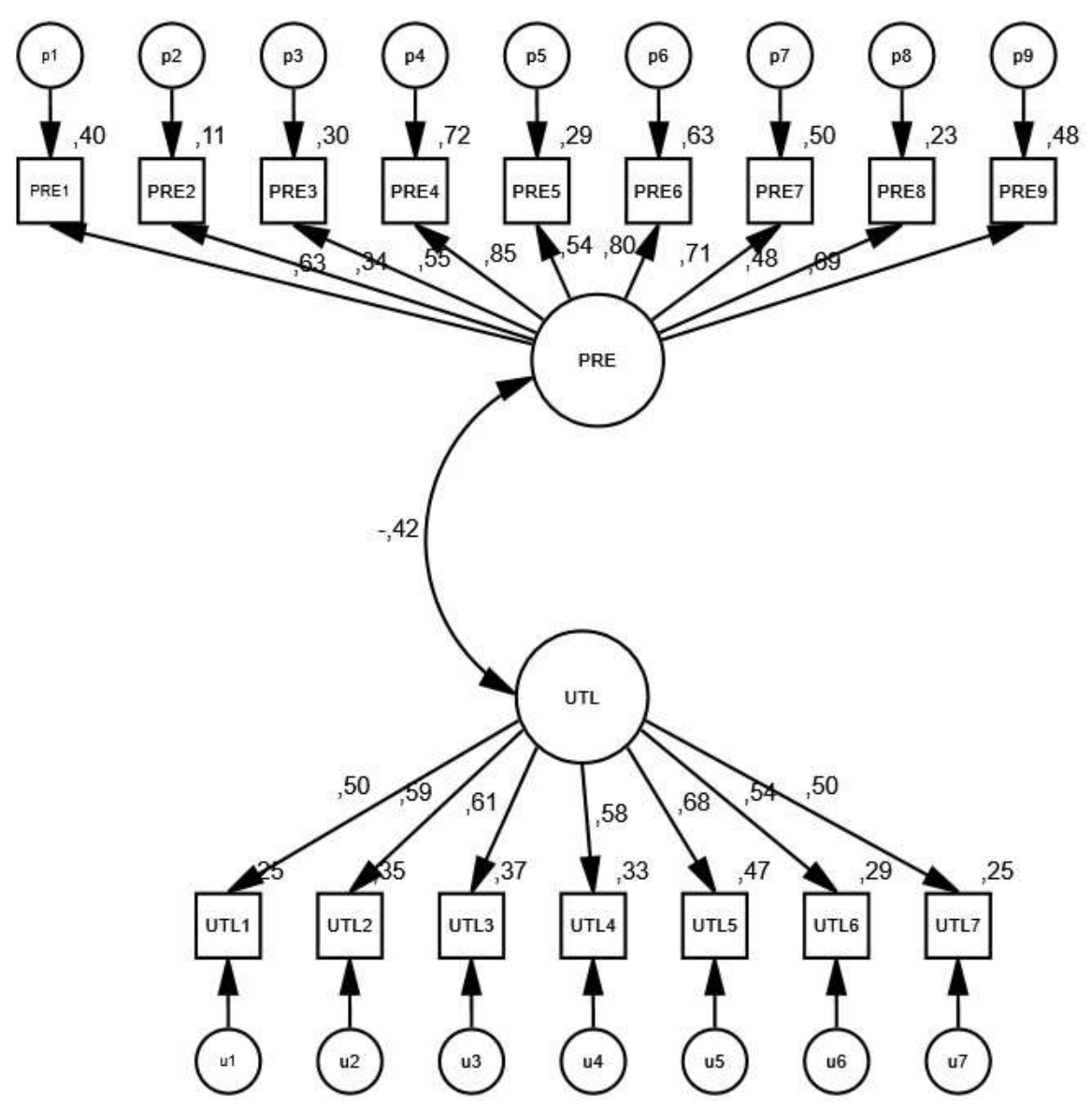

Fonte: Autores.

O modelo estrutural, mostrado na figura 1 , apresentou um ajuste global de $\chi 2 / \mathrm{gl}=2,30$, com o índice da raiz do erro quadrático médio de aproximação (RMSEA=0,088), o índice de qualidade de ajuste (GFI=0,861), e o índice de ajuste comparativo (CFI=0,791). Estes índices estão entre os limites recomendados por Hair et al. (2010) e Byrne (2016), e indicaram o ajuste adequado do modelo de dois fatores.

Desse modo, dois fatores propostos por Johnson e Manoli (2011) e Manoli, Johnson, Buxner e Bogner (2019) emergem de forma clara e consistente na dimensionalidade das escalas. A correlação entre os dois fatores, PRE e UTL, foi de 0,42, explicando em parte que um aumento da percepção de PRE, provoca uma diminuição na percepção de UTL, aumentando o biocentrismo da população.

As frequências dos fatores PRE e UTL da escala 2-MEV são mostradas na Figura 2. Os aspectos visuais dos histogramas indicam que há uma assimetria positiva para PRE e uma assimetria negativa para UTL, podendo considerar que não exista uma normalidade estatísticas nos fatores, caracterizando uma heterocedasticidade, ou seja, a variância dos erros é diferente nos fatores PRE e UTL. 
Figura 2 - Distribuição de frequências para os fatores Preservação (PRE) e Utilização (UTL),
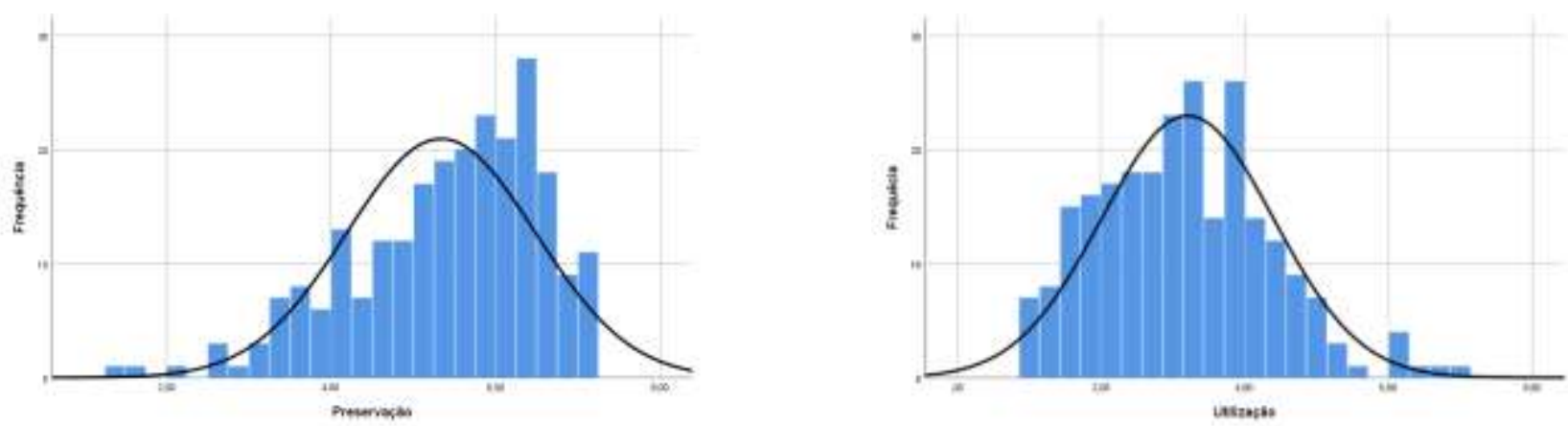

Fonte: Autores.

Com os dados processados dos respondentes, construiu-se a Tabela 2 com as estatísticas descritivas das amostras por área de conhecimento e por quadrante. Os quadrantes PRE- UTL- e PRE - UTL + apresentaram os menores escores médios para o fator PRE, como a menor quantidade de universitários nestes quadrantes.

Tabela 2 - Média e desvio padrão dos escores dos fatores por quadrante do 2-MEV e por Área de conhecimento.

\begin{tabular}{|c|c|c|c|c|c|c|}
\hline \multirow[b]{2}{*}{ Quadrante } & \multirow[b]{2}{*}{ Área do conhecimento } & \multirow[b]{2}{*}{$\mathrm{n}$} & \multicolumn{2}{|c|}{ Preservação } & \multicolumn{2}{|c|}{ Utilização } \\
\hline & & & $\mathrm{m}$ & $\mathrm{dp}$ & $\mathrm{m}$ & $\mathrm{dp}$ \\
\hline \multirow[t]{3}{*}{ PRE+ UTL+ } & Agrárias & 27 & 5,5 & 0,95 & 4,9 & 0,83 \\
\hline & Exatas & 23 & 5,4 & 0,78 & 4,5 & 0,67 \\
\hline & Saúde & 4 & 5,1 & 0,80 & 4,5 & 0,47 \\
\hline \multirow[t]{3}{*}{ PRE + UTL - } & Agrárias & 66 & 5,7 & 0,83 & 2,6 & 0,74 \\
\hline & Exatas & 54 & 5,7 & 0,74 & 2,6 & 0,81 \\
\hline & Saúde & 36 & 5,9 & 0,80 & 2,4 & 0,87 \\
\hline \multirow[t]{3}{*}{ PRE - UTL - } & Agrárias & 4 & 3,0 & 0,70 & 3,3 & 0,55 \\
\hline & Exatas & 15 & 3,3 & 0,63 & 3,2 & 0,42 \\
\hline & Saúde & 1 & 3,6 & . & 2,7 & . \\
\hline \multirow[t]{3}{*}{ PRE- UTL+ } & Agrárias & 6 & 3,4 & 0,51 & 4,4 & 0,39 \\
\hline & Exatas & 4 & 2,8 & 0,97 & 4,8 & 0,91 \\
\hline & Saúde & 1 & 2,5 & . & 4,6 & . \\
\hline \multirow[t]{4}{*}{ Total } & Agrárias & 103 & 5,4 & 1,10 & 3,4 & 1,26 \\
\hline & Exatas & 96 & 5,1 & 1,22 & 3,2 & 1,13 \\
\hline & Saúde & 42 & 5,7 & 1,00 & 2,7 & 1,06 \\
\hline & Total & 241 & 5,3 & 1,15 & 3,2 & 1,20 \\
\hline
\end{tabular}

Legenda: $\mathrm{n}$ - número de respondentes; $\mathrm{m}$ - média; dp - desvio padrão. Fonte: Autores.

Para a melhor identificação da quantidade de universitários nos quadrantes, elaborou-se a Figura 3.

Esta Figura 3 mostra a distribuição das pontuações dos fatores para os quadrantes, considerando os escores individuais de PRE e UTL de cada um dos universitários respondentes, por área de conhecimento. Os símbolos estão descritos na legenda da figura. No quadrante PRE+ UTL- estão localizados os universitários com atitudes mais biocêntrica, considerando os maiores escores no fator PRE e os menores escores no fator UTL. No quadrante PRE- UTL+ estão os universitários com atitudes mais antropocêntricas. Ou seja, com escores maiores no fator UTL e menores no fator PRE. 
Figura 3 - Distribuição dos escores dos participantes para os quadrantes em relação a PRE e UTL e áreas de conhecimento.

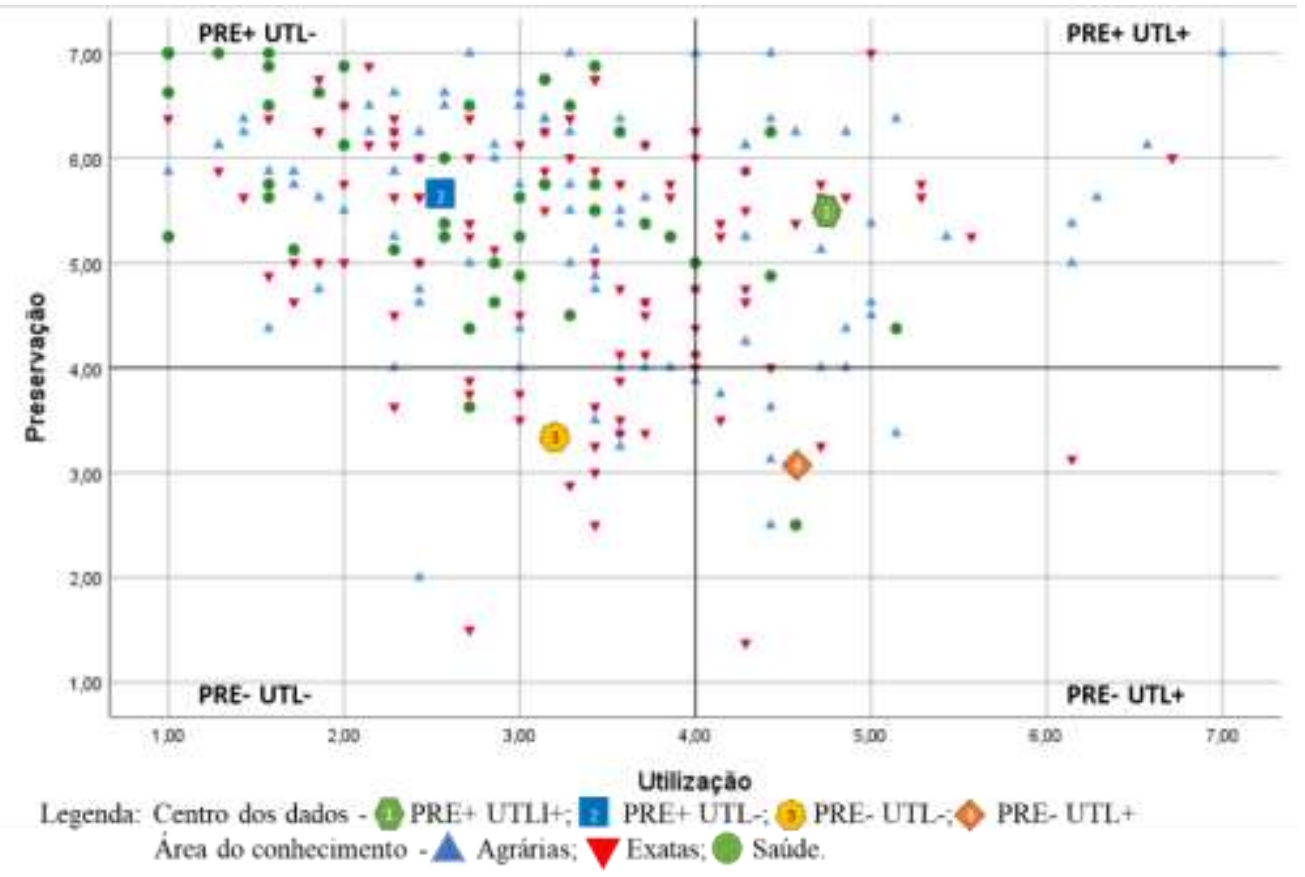

Fonte: Autores.

Por uma análise breve e visual da Figura 4, pode-se perceber que os universitários são a maioria com atitude biocêntrica (quadrante PRE+ UTL-) e a minoria com atitude antropocêntrica (quadrante PRE- UTL+). No quadrante PREUTL- estão localizados os universitários com atitude apática com o meio ambiente, ao expressarem baixos escores nos fatores PRE e UTL, conjuntamente. No quadrante PRE- UTL+ estão localizados os poucos universitários com baixas atitudes conservadoras e com entendimento de que a natureza deve servir aos seres humanos.

Para a interpretação dos perfis dos universitários em relação à PRE e à UTL, foi realizado um estudo de regressões com a PRE como variável dependente e a UTL como variável explicativa. Ressalta-se que a regressão linear simples (RL) considera os seus ajustes em relação aos valores médios dos escores de toda a amostra. Quando os relacionamentos das variáveis são heterocedásticos (relações consideradas na Figura 2), os coeficientes de regressão e as estimativas da variável dependente são médias ruins. Os resultados da regressão quantílica (RQ) mostram diferentes coeficientes e estimativas em diferentes níveis da variável dependente. A RQ permite que se analise o relacionamento entre as variáveis dependente e explicativa para cada quantil da amostra, separadamente. Neste estudo, as RQs foram escolhidas para os quartis $25 \%$ (quartil inferior), $50 \%$ (mediana) e 75\% (quartil superior) dos dados.

A Tabela 3 apresentam os coeficientes não padronizados das variáveis, para toda amostra dos universitários e para cada área do conhecimento.

Analisando a Tabela 3, observou-se que para a RL, o coeficiente de determinação ajustado $\mathrm{R}^{2}$, que indica a proporção da variância de UTL sobre a PRE, apresentam baixos valores de explicação, com um maior valor para a área de conhecimento da Saúde, com 24,8\% da variância explicada em PRE, considerado como valor médio, e as demais, pequenas, pelo critério de Hair Jr et al. (2010). 
Tabela 3 - Regressões linear e quantílicas da variável dependente Preservação em função da variável explicativa Utilização por Área do Conhecimento.

\begin{tabular}{|c|c|c|c|c|}
\hline Variáveis & $\mathbf{R L}$ & RQ25 & RQ50 & RQ75 \\
\hline \multicolumn{5}{|l|}{ Toda amostra } \\
\hline Intercepto & $6,166^{* *}$ & $6,214 * *$ & $6,250 * *$ & $6,804 * *$ \\
\hline Utilização (UTL) & $-0,259 * *$ & $-0,500 * *$ & $-0,219 * *$ & $-0,188 * *$ \\
\hline $\mathbf{R}^{2}$ ajust ou pseudo $\mathbf{R}^{2}$ & 0,069 & 0,066 & 0,038 & 0,031 \\
\hline \multicolumn{5}{|l|}{ Área de conhecimento } \\
\hline \multicolumn{5}{|l|}{ Agrárias } \\
\hline Intercepto & $5,989 * *$ & $6,370^{* *}$ & $6,214 * *$ & $6,444 * *$ \\
\hline Utilização (UTL) & $-0,175^{*}$ & $-0,495 * *$ & $-0,198 \mathrm{~ns}$ & $-0,049 \mathrm{~ns}$ \\
\hline$R^{2}$ ajust ou pseudo $R^{2}$ & 0,031 & 0,066 & 0,042 & 0,004 \\
\hline \multicolumn{5}{|l|}{ Exatas } \\
\hline Intercepto & $5,951 * *$ & $5,654 * *$ & $6,050 * *$ & $6,575 * *$ \\
\hline Utilização (UTL) & $-0,254^{*}$ & $-0,412 *$ & $-0,175 n s$ & $-0,175^{*}$ \\
\hline$R^{2}$ ajust ou pseudo $R^{2}$ & 0,045 & 0,064 & 0,019 & 0,054 \\
\hline \multicolumn{5}{|l|}{ Saúde } \\
\hline Intercepto & $6,986^{* *}$ & $6,214 * *$ & $7,200 * *$ & $7,659 * *$ \\
\hline Utilização (UTL) & $-0,488 * *$ & $-0,375 \mathrm{~ns}$ & $-0,525^{* *}$ & $-0,557^{* * *}$ \\
\hline $\mathbf{R}^{2}$ ajust ou pseudo $\mathbf{R}^{2}$ & 0,248 & 0,100 & 0,180 & 0,152 \\
\hline
\end{tabular}

Legenda: RL - regressão linear; RQ - regressão quantílica para dos quartis 25\%, $50 \%$ e $75 \%$; Nível de significância da estatística $t: *$ p $<0,05$; $* * \mathrm{p}<0,01 ; \mathrm{ns}-\mathrm{p}>0,05$, não significativo. Fonte: Autores.

O pseudo $\mathrm{R}^{2}$, considerada por Koenker e Hallock (2001) como uma estatística de medida de ajustamento local, para cada um dos quartis da distribuição condicional. Com a mudanças da base intervalar para uma base ordinal, os conceitos não serão os mesmos para a porcentagem de variância explicada por $\mathrm{R}^{2}$, que assume um tamanho de efeito homogêneo em todos os níveis da variável dependente. $\mathrm{O}$ pseudo $\mathrm{R}^{2}$ é uma medida da qualidade de ajuste, considerado como coeficiente de determinação ajustada para cada um dos quartis. $\mathrm{Na} R \mathrm{R}$, haverá uma medida pseudo $\mathrm{R}^{2}$ para cada quartil modelado. Pelo critério proposto em Garson (2014), os pseudos $\mathrm{R}^{2}$ são menores que 0,3 , indicando um tamanho do efeito fraco.

Na Tabela 3 são apresentados os coeficientes de regressão dos quartis. A variável UTL tem uma diminuição progressiva nos quartis $25 \%, 50 \%$ e $75 \%$. Para toda a amostra, entende-se que com o aumento da atitude de UTL, há um decréscimo na atitude de PRE ambiental. Considerando o quartil 50\%, quando o universitário indica uma UTL de 1 ponto na escala de Likert de concordância, seu valor será de -0,219 e corresponderá a uma PRE de 6,250 pontos. Enquanto, para um aumento na atitude de UTL de 7 pontos, a PRE diminuirá para 4,7 pontos. A Figura 4 apresenta o diagrama de ajuste dos quantíles para o intercepto e para a variável preditora da UTL. 
Figura 4 - Diagrama da regressão quantílica e regressão linear para os parâmetros estimados, por quantíles.
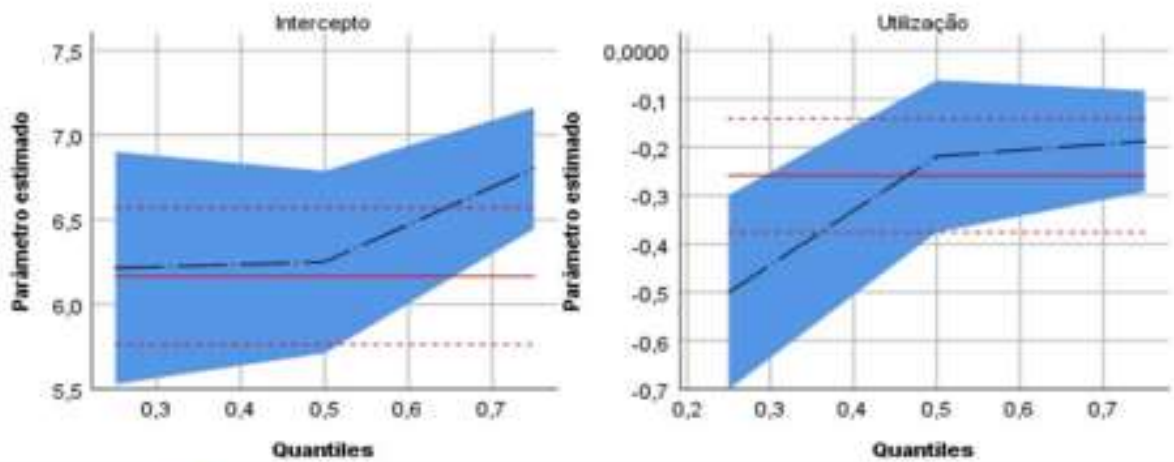
Legenda: Intervalo de confiança a 95\% do parâmetro estimado
-.. Parâmetro estimado por quartil
— Parâmetro estimado pela regressão linear
....... Intervalo de confiança a $95 \%$ da regressão linear

Fonte: dados da pesquisa e os autores.

Observando a Figura 5 pode-se extrair, graficamente, a confirmação dos resultados anteriores. Com o aumento da atitude de UTL há uma diminuição da atitude PRE.

Quando foi estimado se os parâmetros sociodemográficos dos universitários: faixa de idade, semestre cursando e período das aulas; como regressores das atitudes à PRE e à UTL, os resultados dos respectivos coeficientes da RQ não foram significativos a p<0,05. Com isto, inferiu-se que essas categorias não interferem nas atitudes dos universitários em relação à PRE e à UTL da natureza. A Figura 6 fornece as condições de inferir que existem diferenças nas atitudes entre os homens e as mulheres.

Figura 5 - Linhas de predições para a atitude de Preservação em função das categorias feminino e masculino.

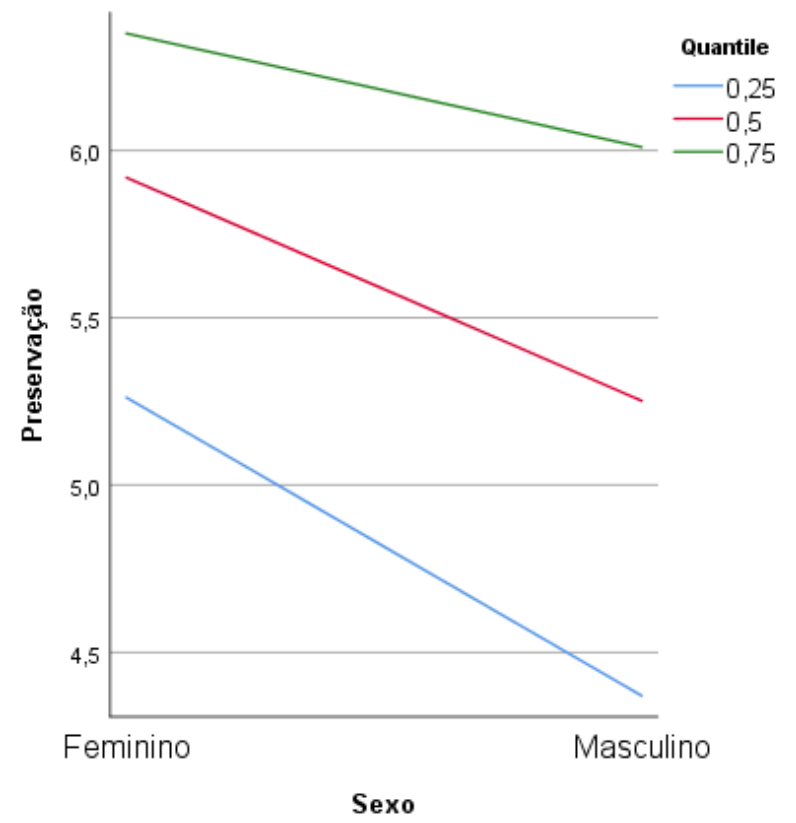

Fonte: Autores. 
Na classificação do universitário quanto ao seu sexo declarado, este mostrou-se significativo a p $<0,05$. As mulheres apareceram com maior atitude biocêntrica do que os homens, nos três quartis avaliados (Figura 5).

Os autores reconhecem que o emprego dos dados provenientes de uma amostra por conveniência, em termos de quantidade de respondentes e de alguns cursos de graduação presencial, dentro outros vários que compoem o portifólio das áreas de conhecimento, pode provocar viés nos resultados generalizados e na restrição de inferências das atitudes de preservação e utilização da natureza mais amplas, para os demais universitários da IES. Recomenda-se que outros estudos sejam realizados ao longo do tempo, testando a modificações das atitudes em função de eventos exôgenos, como também na inserção de programas de educação ambiental, testando a escala antes e depois dos cursos.

Mesmo assim, com os dados consistêntes e significativos, pode-se considerar que os resultados encontrados neste estudo fornecem informações interessantes sobre as atitudes dos universitários, demonstram maneiras atitudinais biocêntritas e antropocêntristas. De tal modo, que valoriza-se o método e os achados deste estudos como uma contribuição expressiva ao conhecimento acadêmico, além de proposcionar o entendimento mais amplo do que se deve realizar em pesquisa futura.

Os autores declaram não haver conflito de interesses e a pesquisa está em conformidade com os padrões éticos. Todos os autores concluíram cooperativamente a conceituação e a redação do presente estudo.

\section{Conclusão}

A proposta da escala 2-MEV mostrou-se consistente na constituição dos dois fatores PRE e UTL, indicando uma correlação negativa. Com a diminuição da UTL há um aumento da PRE. Os universitários da instituição de ensino superior possuem uma atitude preservadora da natureza, ou biocêntrica, destacando-se àqueles universitários da área de conhecimento da saúde. O modelo de predição das relações entre PRE e UTL são consistentes e analíticos com o emprego da RQ. No efeito de moderação pelas características sociodemográficas dos universitários entre PRE e UTL, nos quartis, apenas existem diferenças entre os homens e as mulheres. Os homens são mais antropocêntricos e as mulheres mais biocêntricas.

Recomenda-se, como sugestões para estudos futuros, que eles sejam realizados ao longo do tempo, como uma pesquisa longitudinal, testando a modificações das atitudes em função de eventos exógenos, como também, após a inserção de programas de educação ambiental, esta escala seja testada para avaliar as atitudes declaradas antes e depois dos cursos.

\section{Agradecimentos}

Os autores agradecem aos Programas de Pós Graduação Stricto Sensu da UNIDERP pelo suporte financeiro e de infraestrutura para a realização desta pesquisa. Também, o nosso reconhecimento aos dois referees anônimos pelos seus comentários, sugestões e ajustes, adequados e criteriosos.

\section{Referências}

Ajzen, I. (2020). The theory of planned behavior: Frequently asked question. Human Behavior and Emerging Technologies, 2(4), 314-324. doi: $10.1002 / \mathrm{hbe} 2.195$

Ajzen, I., \& Fishbein, M. (1977). Attitude-behavior relations: A theoretical analysis and review of empirical research. Psychological Bulletin, 84(5), 888-918. Arbuckle, J. L. (2016). IBM SPSS AMOS 24 User's Guide. IBM.

Blaikie, N. W. (1992). The nature and origins of ecological world views: An Australian study. Study. Social Science Quarterly, 73(1), 144-165.

Bogner, F. X. (2018). Environmental values (2-MEV) and appreciation of nature. Sustainability, 10(2), 350. 10.3390/su10020350

Bogner, F. X., \& Wiseman, M. (2006). Adolescents' attitudes toward nature and environment: Quantifying the 2-MEV model. Environmentalist, 26(4), 247254. 10.1007/s10669-006-8660-9 
Boucher, Y., Arseneault, D., Sirois, L., \& Blais, L. (2009). Logging pattern and landscape changes over the last century at b oreal and dciduous forest transition in Eastern Canada. Landscape Ecology, 24(2), 171-184.

Byrne, B. M. (2016). Structural Equation Modeling with Amos (3th ed.). Routledge.

Costa, L. F., Neumann, S. E., Dorion, E. C., Olea, P., \& Severo, E. A. (2019). Sustentabilidade e Desenvolvimento Sustentávelno Contexto das Ciências Sociais: do século XVIII ao século XXI. Revista Metropolitana de sustentabilidade, 9(2), 6-19.

Dunlap, R. E., \& Van Liere, K. D. (1978). The New Environmental Paradigm. Journal of Environmental Education, 9(4), 10-19. doi:10.1080/00958964.1978.10801875

Dunlap, R. E., Van Liere, K. D., Mertig, A. G., \& Jones, R. E. (2000). Mensuring endorsment of the New Ecological Paradigm: A revised NEP Scale. Journal of Social Issues, 56(3), 425-442.

Faul, F., Erdfelder, E., Lang, A. G., \& Buchener, A. (2007). G*Power 3: A flexible statisitical power analysis program for social, behavioral, and biomedical sciences. Behavior research methods, 39(2), 175-191.

Foley, J. A., DeFries, R., Asner, G. P., Barford, C., \& Bonan, G. e. (2005). Global consequences of land use. Science, 309(5734), 570-574. $10.1126 /$ science. 1111772

Garson, G. D. (2014). Multiple Regression. Asheboro, NC: Statistical Associates Publishing.

Gerhardt, T. E., \& Silveira, D. T. (2009). Métodos de Pesquisa. Editora da UFRGS.

Hair Jr, J. F., Black, W. C., Babin, B. J., \& Anderson, R. E. (2010). Multivariate Data Analysis (7th ed.). Prentice Hall.

Johnson, B., \& Manoli, C. C. (2011). The 2-MEV scale in United States: A measure of clildren's environmental attitudes based on the theory of ecological attitude. The Journal of Environmental Education, 42(2), 84-97. 10.1080/00958964.2010.503716

Koenker, R., \& Hallock, K. (2001). Quantile Regression. Journal of Economic Perspectives, 143-156.

Kopnina, H. (2013). Evaluating education for sustainable development (ESD): using Ecocentric and Anthropocentric Attitudes Toward the Sustainable Development (EAATSD) scale. Environment, Development and Sustainability, 15, 607-623.10.1007/s10668-012-9395-z

Manoli, C. C., Johnson, B., Buxner, S., \& Bogner, F. (2019). Measuring environmental perceptions groundede on different theoretical models: The 2-Major Environmental Values (2-MEV) model in comparison with New Ecological Paradigm (NEP) scale. Sustainability, 11(5), p. 1286. doi:10.3390/su11051286

Memon, M. A., Ting, H., Cheah, J. H., Thurasamy, R., Chuah, F., \& Cham, T. H. (2020, June). Sample size for survey research: Review and recomendations. Journal of Applied Structural Equation Modeling, 4(2), i-xx. 10.47263/JASEM.4(2)01

Mikhailova, I. (2004). Sustentabilidade: evolução dos conceitos teóricos e os problemas de mensuração prática. Revista Econômia e Desenvolvimento, 16, 2241 .

Nossa, V., Rodrigues, V. R., \& Nossa, S. N. (2017). O que se tem pesquisado sobre Sustentabilidade Empresarila e sua Evidenciação? Revista de Educação e Pesquisa em Contabilidade, 11, 87-105.10.17524/repec.v11i0.1719

Pereira, A. S., Shitsuka, D. M., Parreira, F. J., \& Shitsuka, R. (2018). Metodologia da pesquisa científica. UAB/NTE/UFSM. https://repositorio. ufsm. br/bitstream/handle/1/15824/Lic_Computacao_Metodologia-Pesquisa-Cientifica. pdf.

Sachs, I. (2008). Desenvolvimento: includente, sustentável e sustentado. Garamond.

Sarstedt, M., \& Mooi, E. (2018). A concise guide to market research: the process, data, and methods using IBM SPSS Statistics. Springer. 